\title{
Resectable Rectal Cancer: Which Patient Does Not Need Preoperative Radiotherapy?
}

\author{
Yakup Kulu Alexis Ulrich Markus W. Büchler \\ Department of General, Visceral and Transplantation Surgery, University of Heidelberg, Heidelberg, Germany
}

\section{Key Words}

Rectal cancer • Preoperative radiotherapy •

Chemoradiotherapy $\cdot$ Neoadjuvant treatment $\cdot$ Local control

\begin{abstract}
It is well known that some patients with resectable rectal cancer benefit from preoperative radiotherapy in combination with or without chemotherapy. In order to reduce local recurrence and improve long-term survival, current guidelines advocate such neoadjuvant treatment in UICC (Union for International Cancer Control) stage II and III patients. However, the vast majority of patients may be adequately treated by rectal resection with total mesorectal excision (TME) alone. Recent evidence suggests an overtreatment of patients leading to unnecessary exposure to acute and longterm toxicity of radiation therapy. The question which consequently arises is which patient does not need preoperative radiotherapy. Improvements in MRI combined with better understanding of prognostic indicators suggest that patients with UICC stage I tumors, with tumors more than $12 \mathrm{~cm}$ proximal the anal verge can and patients with a circumferential resection margin $\geq 2 \mathrm{~mm}$ as assessed by preoperative MRI might be managed by radical surgery with adequate TME alone.

Copyright $\odot 2012$ S. Karger AG, Basel
\end{abstract}

\section{Introduction}

Colorectal cancer is a common malignancy and ranks as the 3rd most common cancer in both genders worldwide. In 2011 it was estimated that more than 160,000 patients died of colorectal cancer in Europe [1]. Early reports of local recurrence rates after curative resections of rectal cancer were as high as $38 \%[2,3]$. In the last few decades, improvements in surgical technique have resulted in local recurrence rates less than $8 \%[4,5]$. Today, the entire removal of the mesorectum by sharp dissection under visual control along the visceral fascia of the mesorectum and the parietal fascia of the pelvis is accepted as the standard approach in rectal cancer surgery and is referred to as total mesorectal excision (TME).

Considerable debate has evolved about the significance of preoperative radiotherapy with or without chemotherapy in the treatment of resectable rectal cancer. Two meta-analyses evaluated the benefit of neoadjuvant radiotherapy $[6,7]$. They concluded that preoperative radiation therapy was associated with significantly less local recurrence. In the CAO/ARO/A10-94 trial, patients with stage II and III rectal cancer were randomly assigned to receive either preoperative or postoperative chemoradiotherapy [8]. Preoperative chemoradiotherapy was associated with improved local control and less toxic-

\section{KARGER}

Fax +41613061234

E-Mail karger@karger.ch

www.karger.com
(C) 2012 S. Karger AG, Basel

$0257-2753 / 12 / 0308-0118 \$ 38.00 / 0$

Accessible online at:

www.karger.com/ddi
Markus W. Büchler, MD

Department of General, Visceral and Transplantation Surgery

University of Heidelberg, Im Neuenheimer Feld 110

DE-69120 Heidelberg (Germany)

Tel. +496221 566 201, E-Mail markus.buechler@med.uni-heidelberg.de 
Table 1. Dutch trial [18]

\begin{tabular}{|c|c|c|c|c|c|c|c|}
\hline & \multirow[t]{2}{*}{$\mathrm{n}$} & \multicolumn{2}{|c|}{ 10-year LR, \% } & \multirow[t]{2}{*}{$\mathrm{p}$} & \multicolumn{2}{|c|}{10 -year OS, \% } & \multirow[t]{2}{*}{$\mathrm{p}$} \\
\hline & & $\mathrm{RT}+\mathrm{TME}$ & TME & & $\mathrm{RT}+\mathrm{TME}$ & TME & \\
\hline All patients & 1,748 & 5 & 11 & $<0.0001$ & 56 & 57 & 0.88 \\
\hline TNM I & 507 & $<1$ & 3 & 0.027 & 65 & 72 & 0.321 \\
\hline TNM II & 491 & 5 & 8 & 0.212 & 50 & 55 & 0.242 \\
\hline TNM III & 622 & 9 & 19 & $<0.0001$ & 39 & 37 & 0.526 \\
\hline \multicolumn{8}{|c|}{ CRM-negative } \\
\hline TNM I & 497 & $<1$ & 3 & 0.027 & 65 & 72 & 0.293 \\
\hline TNM II & 421 & 4 & 7 & 0.355 & 51 & 57 & 0.213 \\
\hline TNM III & 435 & 5 & 17 & $<0.0001$ & 50 & 40 & 0.032 \\
\hline
\end{tabular}

$\mathrm{RT}=$ Radiotherapy; $\mathrm{LR}=$ local recurrence; $\mathrm{OS}=$ overall survival.

ity. Furthermore, a statistically significant increase in sphincter preservation was achieved among patients after preoperative chemoradiation. Based on these results, guidelines in Germany advocate that rectal cancer patients with stage II and III disease after preoperative staging be given neoadjuvant radiotherapy or chemoradiotherapy [9].

However, the current classification system of rectal cancer patients as stage II and stage III has limitations. Patients with T3 tumors incorporate a heterogeneous group of patients with markedly different prognoses depending on the extent of extramural tumor spread [1012]. For the patient who is selected to receive neoadjuvant radiotherapy, the expected benefit and harm has to be well balanced. Side effects of radiotherapy such as fecal incontinence, sexual dysfunction, bowel dysfunction, and secondary malignancy can impair quality of life and shorten life expectancy [13-18]. Routine, nonselective neoadjuvant treatment also has major financial implications for health care providers. Recent improvements in preoperative staging may offer possibilities to carefully identify the individual patient who does not need preoperative radiotherapy.

\section{Background}

The following large randomized controlled studies in the TME era evaluated preoperative or postoperative radiation therapy with or without chemotherapy.

A Dutch trial randomly assigned 1861 patients with resectable rectal cancer to preoperative radiotherapy with $5 \times 5$ Gy followed by TME within a week or to TME alone [18]. The 10-year cumulative incidence of local recurrence was significantly reduced by radiotherapy compared to surgery alone ( 5 vs. $11 \%, \mathrm{p}<0.0001$ ). Although patients without neoadjuvant treatment died more often from cancer, pretreated patients presented other causes of death - especially secondary carcinomas within the radiation field - resulting in an equal overall survival in both groups. In a subgroup of patients with negative circumferential resection margin (CRM), only the patients with stage III disease benefited from preoperative radiotherapy, possibly underscoring the relevance of neoadjuvant radiotherapy in patients with positive nodal status. However, it has to be taken in consideration that the CRM was evaluated by the pathologist after the operation and not by MRI preoperatively [19]. Furthermore, the quality of TME was completely inadequate in $24 \%$ of the patients [19], increasing the risk of local recurrence especially in stage III disease. In contrast, patients with negative circumferential margin (CRM) and stage I or II disease did not benefit from additional preoperative radiotherapy (table 1).

Another recently published multicenter randomized trial randomly assigned 1,350 patients to neoadjuvant short-term radiotherapy or selective postoperative chemoradiotherapy [20]. Patients were selected to receive either neoadjuvant treatment independent of tumor stage or postoperative chemoradiotherapy, if the histopathological CRM was involved in the group not receiving neoadjuvant treatment. Overall survival did not differ between groups. However, after 5 years, the local recurrence rate of patients following neoadjuvant irradiation was significantly reduced compared to patients after selective chemoradiotherapy ( 4.7 vs. $11.5 \%, \mathrm{p}<0.001$ ). 
Sauer et al. [8] demonstrated in their multicenter randomized trial involving 823 patients a significant reduction in local recurrence and late toxic effects in patients with neoadjuvant chemoradiotherapy compared to postoperative chemoradiotherapy. After 5 years, the local recurrence rate in patients after preoperative chemoradiotherapy was reported to be $6 \%$, compared to $13 \%$ in the group assigned to postoperative treatment ( $p>0.006$ ). Acute and late toxic grade 3 or 4 effects were significantly more frequent in patients receiving postoperative chemoradiotherapy (40 vs. $27 \%, \mathrm{p}<0.001 ; 24$ vs. $14 \%$, $\mathrm{p}>0.01)$. Despite starting systemic treatment preoperatively, no statistically significant difference, neither in distant recurrence nor in disease-free or overall survival, could be demonstrated. Following preoperative staging evaluation, patients with stage II and III rectal cancer were recruited. Histopathological examination revealed that $8 \%$ of the patients assigned to the preoperative group became complete responders and $25 \%$ of the patients assigned to the preoperative group were stage I. This downstaging can be attributed to the preoperative chemoradiotherapy. However, $18 \%$ of the patients assigned to the postoperative chemoradiotherapy group were initially overstaged since histopathological examination revealed stage I disease.

\section{Radiotherapy and Its Side Effects}

Radiotherapy can be associated with considerable side effects. The patient collective participating in the Dutch study was evaluated for late side effects of short-course preoperative radiotherapy [17]. In patients without a stoma, mean bowel frequency during the day was significantly higher in irradiated patients compared to patients after surgery alone. The evaluated variables to determine the bowel function were fecal incontinence during the day and at night, anal blood and mucus loss, and higher rates of pad wearing. Irradiated patients were affected significantly more frequently by the reported variables. Other factors, such as impact of bowel function on daily activities or overall satisfaction with bowel function, were more frequently reported by irradiated patients, compared to surgery-alone patients, but this difference did not reach statistical significance. No difference in satisfaction between the two groups was noted in patients with a stoma.

Bruheim et al. [15] evaluated the sexual function in males after radiotherapy for rectal cancer. Questionnaires were returned from 241 patients (108 patients after
Table 2. Effect of plane of surgery achieved on 3-year local recurrence (\%) [20]

\begin{tabular}{llll}
\hline & $\begin{array}{l}\text { Muscularis } \\
\text { propria }\end{array}$ & $\begin{array}{l}\text { Intra- } \\
\text { mesorectal }\end{array}$ & $\begin{array}{l}\text { Meso- } \\
\text { rectal }\end{array}$ \\
\hline $\begin{array}{l}\text { Overall } \\
\text { Treatment }\end{array}$ & 13 & 7 & 4 \\
$\quad$ Preoperative RT & 10 & 4 & 1 \\
$\quad$ Selective postop. CRT & 16 & 10 & 7 \\
TNM stage & & & \\
$\quad$ I & 8 & 2 & 0 \\
$\quad$ II & 6 & 2 & 5 \\
$\quad$ III & 20 & 14 & 6 \\
\hline
\end{tabular}

$\mathrm{RT}=$ Radiotherapy CRT $=$ chemoradiotherapy.

preoperative or postoperative (chemo)radiotherapy and 133 patients without radiotherapy) a median of 4.5 years after rectal cancer surgery. Irradiated patients had significantly poorer scores for erectile function, orgasmic function, intercourse satisfaction, and overall satisfaction with sex life compared with nonirradiated patients. Irradiated patients more often had pT4 tumors, more frequent tumors closer to the anal verge, and more frequent abdominoperineal resections compared to the nonirradiated patients. However, even after exclusion of patients with prostate, seminal vesicle, and pelvic floor resections, sexual function of irradiated patients remained poorer compared to nonirradiated patients.

Radiotherapy causes additional long-term side effects and may impair quality of life. Besides that, radiotherapy may shorten life expectancy by causing secondary malignancies. Secondary malignancy was reported to be the cause of death in $14 \%$ of patients after preoperative radiotherapy prior to TME, compared to $9 \%$ after TME alone and a median follow-up of 10 years in the Dutch trial [18].

\section{Quality of Surgery}

Surgery has been the cornerstone of treatment for patients with resectable rectal cancer. After the introduction of TME by Heald and Ryall [5], local recurrence after rectal cancer surgery dropped below $8 \%$ [21]. Training in surgical technique to perform TME improved oncological outcome in populations from Norway, Sweden, and the Netherlands [22-24]. Quirke et al. [25] evaluated the involvement of the CRM and the quality of TME achieved in 1,156 patients with resectable rectal cancer from the 
Table 3. Oncological outcome based on tumor location and TNM staging

\begin{tabular}{|c|c|c|c|c|c|c|c|}
\hline Trial, year & Study design & LR, \% & $\mathrm{p}$ & LR, \% & $\mathrm{p}$ & LR, \% & $\mathrm{p}$ \\
\hline Tumor location & & $<5 \mathrm{~cm}$ & & $5-10 \mathrm{~cm}$ & & $>10 \mathrm{~cm}$ & \\
\hline Dutch study 2011 [18] & RT vs. TME & 10.7 vs. 12 & 0.578 & 3.7 vs. 13.7 & $<0.0001$ & 3.7 vs. 6.2 & 0.122 \\
\hline SRCT 1997 [37] & RT vs. OP & 10 vs. 27 & 0.003 & 9 vs. 26 & $<0.0001$ & 8 vs. 12 & 0.3 \\
\hline TNM Staging & & UICC I & & UICC II & & UICC III & \\
\hline Dutch study 2011 & RT vs. TME & 0.4 vs. 14 & 0.091 & 5.3 vs. 7.2 & 0.331 & 10.6 vs. 20.6 & $<0.0001$ \\
\hline SRCT 1997 & RT vs. OP & 4.5 vs. 14 & 0.009 & 6 vs. 22 & $<0.0001$ & 23 vs. 46 & $<0.0001$ \\
\hline
\end{tabular}

LR = Local recurrence; $\mathrm{RT}=$ radiotherapy; OP = operation; SRCT = Swedish rectal cancer study; UICC = Union for International Cancer Control.

MRC CR07 trial. The quality of TME was strongly associated with local recurrence. Local recurrence rates after 3 years increased depending on the plane of surgery achieved from $4 \%$ if it was within the mesorectal plane, to $7 \%$ if it was within the intramesorectal plane, up to $13 \%$ if the resection plane was within the muscularis propria (log-rank $p=0.0039$; table 2). In the MRC CR07 trial, the use of TME was not mandated in the trial protocol. Consequently, surgery within the mesorectal plane was achieved in only $52 \%$ of the patients. This suggests that a further decrease in local recurrence rates might be obtained by improving the surgical plane achieved (table 2).

\section{Tumor Height}

There is still debate about the necessity to radiate patients with tumors of the upper third of the rectum. In the United States, high rectal cancers are treated like colon cancer, whereas in many European countries, including Germany, pretreatment is advocated. However, a subgroup analysis of the Dutch study revealed no significant reduction of local recurrence rate by radiotherapy in patients with rectal cancer more than $10 \mathrm{~cm}$ from the anal verge [18]. The same is true for the patients treated within the Swedish Rectal Cancer Trial [26]. Even not operated following the TME principles, patients with tumors more than $11 \mathrm{~cm}$ from the anal verge had no significant reduction of local recurrence if additionally subjected to preoperative radiotherapy (table 3). Nowadays, the majority of colorectal surgeons consider $12 \mathrm{~cm}$ from the anal verge as the beginning of the upper third of the rectum. Due to the limited effect of neoadjuvant treatment in high rectal tumors, there is a tendency to omit neoadjuvant treatment.

\section{TNM Stage}

In the Dutch trial comparing short-term radiotherapy followed by TME with TME alone, no significant differences were found for stage I and II disease in terms of local recurrence and overall survival after 10 years [18]. In contrast, patients with stage III disease and neoadjuvant treatment showed a significantly reduced rate of local recurrence after 10 years ( 9 vs. 19\%, p < 0.0001), though without survival benefit (10-year overall survival: 39 vs. $37 \%, \mathrm{p}=0.526$ ) [18]. Only in a further subgroup analysis of patients with stage III disease and negative CRM could a significant survival benefit of $10 \%$ over 10 years be detected. The disadvantage of the postoperative evaluation of the CRM compared to preoperative evaluation by MRI has been mentioned before. Interestingly, the same subgroup analysis for stage I disease revealed a statistically significant risk reduction of $2.6 \%$ for local recurrence for patients with stage I tumors if subjected to neoadjuvant radiotherapy. It is common sense that patients with stage I disease should not undergo neoadjuvant treatment, displaying the difficulties in the interpretation of subgroup analyses of further subgroup analyses. Consequently, the authors concluded that an absolute risk reduction of $2.6 \%$ for all eligible patients implies that 38 patients have to be exposed to radiotherapy to save 1 patient a local recurrence without any survival benefit (table 1).

\section{Lymph Nodes Involved}

The relevance of nodal status has been highlighted in a study by Hermanek et al. [27]. They evaluated the relationship between the number of involved regional lymph nodes and the risk of locoregional recurrence. While there was no statistically significant difference in local 
recurrence between $\mathrm{pN} 0$ and $\mathrm{pN} 1$, there were highly significant differences $(\mathrm{p}<0.001)$ between $\mathrm{pN} 1$ and $\mathrm{pN} 2$. In patients with $\mathrm{pN} 2$ nodal status, the rate of local recurrence was about $30 \%$. The authors concluded that in this patient collective, neoadjuvant treatment is indicated. However, preoperative lymph node assessment with the diagnostic tools available to date still lacks accuracy with a positive predictive value of about $60 \%$. New diagnostic modalities to predict the presence of lymph nodes involved are clearly warranted.

\section{Circumferential Margin}

Another important variable to enable the preoperative differentiation of patients with good versus poor prognosis tumors is the determination of the circumferential tumor margin involvement [28]. In a recent work by Nagtegaal and Quirke [29], the relevance of CRM was evaluated by reviewing the published data of 17,568 rectal cancer patients. The authors concluded that the power of the CRM to predict local recurrence and, to a lesser extent, development of distant metastases and survival is high, and that CRM involvement is one of the strongest prognosticators.

In 1986, Quirke et al. [30] demonstrated in their histopathological study the relevance of the CRM. Thorough examination of operative specimens of 52 patients revealed that the sensitivity, specificity, and positive predictive value in predicting local recurrence were 92, 95, and $85 \%$, respectively.

Nonirradiated patients of the Dutch study were analyzed for CRM involvement and oncologic outcome [19]. After a median follow-up of 35 months, a CRM of $\leq 2$ $\mathrm{mm}$ was associated with a local recurrence risk of $16 \%$ compared with $5.8 \%$ in patients with a CRM of $\geq 2 \mathrm{~mm}$ ( $p<0.0001)$. Within the first 2 years of follow-up, 37.6\% of the patients with a CRM of $\leq 1 \mathrm{~mm}$ developed distal metastases, whereas patients with a CRM of $\geq 1 \mathrm{~mm}$ metastasized significantly less frequently $(12.7 \%, \mathrm{p}<$ 0.0001). The relevance of CRM was also evaluated in patients who participated in the MRC CR07 study. Quirke et al. [25] assessed 1,156 patients and found that negative CRM was associated with a significantly reduced local recurrence rate (negative CRM vs. positive CRM: 6 vs. $17 \%)$. The plane of surgery achieved was strongly associated with local recurrence. Subgroup analysis revealed that local recurrence rates after 3 years in patients with a stage III tumor was $20 \%$ if the surgical plane achieved was the muscularis propria, $14 \%$ in case of intramesorectal, and as low as $6 \%$ if the surgical plane achieved was the mesorectum.

In addition to the above-mentioned evidence on the significance of CRM, extramural tumor spread influences prognosis [12,31-33]. Rectal cancers invading through the muscularis propria into subserosa or into nonperitonealized pericolic or perirectal tissues not directly invading other organs or structures nor perforating the visceral peritoneum are termed pT3. Merkel et al. [11] evaluated 1,453 patients with $\mathrm{pT} 3$ rectal carcinomas who were treated by radical surgery alone and assessed for oncological outcome depending on extramural spread. Patients with rectal cancer with a maximal tumor invasion beyond the outer border of the muscularis propria of up to $5 \mathrm{~mm}$ were categorized as pT3a, and more than $5 \mathrm{~mm}$ were categorized as pT3b. Local recurrence after 5 years was significantly less frequent in patients with $\mathrm{pT} 3 \mathrm{a}$ rectal cancer, compared to patients with pT3b (10.4 vs. $26.3 \%$, $\mathrm{p}<0.0001)$. The cancer-related 5 -year survival rates were $85.4 \%$ for $\mathrm{pT} 3 \mathrm{a}$ and $54.1 \%$ for $\mathrm{pT} 3 \mathrm{~b}$ ( $\mathrm{p}<0.0001)$. Comparing the oncological outcome of patients with pT2 and pT4 rectal cancer, no significant differences concerning local recurrence and cancer-related survival were observed between $\mathrm{pT} 2$ versus $\mathrm{pT} 3 \mathrm{a}$ and $\mathrm{pT} 3 \mathrm{~b}$ versus $\mathrm{pT} 4$. Careful preoperative determination of extramural spread might identify the subgroup of patients with stage II rectal cancer (pT3apTN0) not in need of neoadjuvant treatment.

The quintessence of the above-mentioned histopathological studies may well be that the benefit from preoperative treatment for patients who have tumors with less than $5 \mathrm{~mm}$ of extramural tumor spread is going to be minimal. In contrast to that, preoperatively identified patients with tumors with extensive tumor spread would most likely benefit from additional preoperative treatment.

To demonstrate the feasibility of preoperative determination of extramural tumor spread via thin sectioning MRI, a prospective multicenter study was initiated. The Magnetic Resonance Imaging and Rectal Cancer European Equivalence (MERCURY) study prospectively evaluated the accuracy of MRI in depicting the extramural spread of tumor invasion of patients with rectal cancer [28]. The preoperative assessment was correlated with histopathological analysis. Evaluation of 295 patients with preoperative magnetic resonance and histopathological measurements revealed a mean extramural depth of invasion at MRI of $2.8 \mathrm{~mm} \pm 4.6$ and at histopathologic examination of $2.81 \mathrm{~mm} \pm 4.28$. Therefore, magnetic resonance and histopathologic assessment of tumor spread were considered equivalent to within $0.5 \mathrm{~mm}$. 
Table 4. Oncological outcome of recently reported series based on AJCC staging

\begin{tabular}{lllll}
\hline & $\begin{array}{l}\text { Treatment } \\
\text { received }\end{array}$ & $\begin{array}{l}\text { LR } \\
\%\end{array}$ & $\begin{array}{l}\text { OS } \\
\%\end{array}$ & $\begin{array}{l}\text { Follow-up } \\
\text { years }\end{array}$ \\
\hline Stage II & & & & \\
MERCURY [35] & TME & 2.3 & 65.7 & 5 \\
MRC CR07 [20] & pre-RT + TME & 1.9 & NA & 3 \\
& TME + post-CRT & 6.4 & NA & 3 \\
Dutch study [18] & TME & 8 & 55 & 10 \\
& pre-RT + TME & 5 & 50 & 10 \\
\hline Stage III & & & & \\
MERCURY & TME & 0 & 81 & 5 \\
MRC CR07 & pre-RT + TME & 7.4 & NA & 3 \\
& TME + post-CRT & 15.4 & NA & 3 \\
Dutch study & TME & 17 & 40 & 10 \\
& pre-RT + TME & 5 & 50 & 10 \\
\hline
\end{tabular}

$\mathrm{LR}=$ Local recurrence; $\mathrm{OS}$ = overall survival; pre-RT = preoperative radiotherapy; post-CRT $=$ postoperative chemoradiotherapy.

\section{Preoperative Staging and Radiotherapy}

Simunovic et al. [34] demonstrated in their institutional study of 150 patients that with preoperative clinical assessment of rectal cancer, preoperative irradiation can be spared in the majority of the patients. Based on assessment of tumor size, fixation, and distance from anal verge, 115 patients ( $42.6 \%$ stage I/II, $33.9 \%$ stage III, and $23.5 \%$ stage IV) were subjected to surgical resection with TME. After a median follow-up of 29 months, the local recurrence rate was $2.6 \%$. Patients deemed to be at high risk of recurrence received radiotherapy. In this group the local recurrence rate was $17.1 \%$.

In the framework of the MERCURY study, 122 patients were identified with rectal cancers defined as good prognosis tumor [tumor $>1 \mathrm{~mm}$ to the mesorectal fascia, no evidence of extramural venous invasion on MRI, T1/ T2, T3a (less than $5 \mathrm{~mm}$ extramural spread)] [35]. None of the patients received preoperative or postoperative radiotherapy. The overall local recurrence after 5 years was $3.3 \%$ (table 4).

Frasson et al. [36] assessed the factors that predict recurrence in patients with cT3 and cT2N+ rectal cancer, staged preoperatively by endorectal ultrasonography and/or MRI. Threatened mesorectal fascia (free margin $\leq 2 \mathrm{~mm}$ ) at preoperative staging was the only significant independent preoperative factor for oncological outcome. Patients who had a threatened CRM or the CRM was not determined preoperatively had a higher risk for local recurrence (19.4 vs. $5.4 \%, \mathrm{p}=0.07)$, shorter disease-free survival (52.7 vs. $69.4 \%, \mathrm{p}=0.07)$ and shorter cancer-specific survival (64.0 vs. $82.3 \%, \mathrm{p}=0.05$ ) compared to patients with preoperatively evaluated negative CRM after 5 years.

\section{Discussion}

Several clinical trials were conducted to find out how to improve oncologic outcome of rectal cancer patients. Before TME was introduced, local recurrence after surgery alone was high. The Swedish Rectal Cancer Trial demonstrated a statistically significant reduction in local recurrence rate for patients after preoperative radiotherapy compared to surgery alone (11 vs. $27 \%, \mathrm{p}<0.001$ ), and a favorable overall survival benefit ( 58 vs. $48 \%, \mathrm{p}<0.004$ ) $[26,37]$. Since surgery has been carried out within the correct anatomical layers, the role of radiotherapy with or without chemotherapy has been questioned.

Patient selection is key. The current guidelines indicating neoadjuvant treatment for all patients with stage II and III rectal cancer have to be challenged. Based on the currently available data, 95 patients have to be radiated without any benefit to avoid local recurrence in 5 patients. At the same time, the treatment has no positive impact on overall survival, in part due to secondary carcinomas induced by radiotherapy. Furthermore, many irradiated patients have to suffer from side effects like impaired stool and sexual function. Therefore, randomized trials have to be conducted to better identify patients benefitting from pretreatment. Simunovic et al. [34] and the MERCURY Study Group have shown that the preoperative evaluation of the CRM by MRI could help to improve the selection of patients. In case of an anticipated negative CRM, neoadjuvant treatment might not be necessary. Randomized controlled trials have to be conducted to investigate this hypothesis.

That preoperative treatment for patients with locally advanced rectal cancer which invades neighboring anatomical structures is part of the multimodal therapy is out of question.

Controversy still exists concerning the efficacy of radiation in high rectal cancers above $12 \mathrm{~cm}$ from the anal verge. Preoperative radiotherapy could not reduce the rate of local recurrence or improve survival in these patients in the Dutch trial including TME $[35,37]$.

If the surgeon follows the principles of TME, the relevance of the nodal status alone is limited. There is suspicion that patients with $\mathrm{pN} 2$ have a greater risk of local 
recurrence; however, lymph node assessment during preoperative staging lacks accuracy [27]. On the other hand, Cecil et al. [38] demonstrated that TME allows resection of all mesorectal lymph nodes, resulting in a favorable oncologic outcome, even without preoperative radiotherapy. It is difficult to understand why the lymph node status should play such an important role, if an adequate TME was performed, the preoperative CRM was negative and, therefore, the perirectal lymph nodes are resected completely. However, lateral lymph nodes, affected by the neoadjuvant treatment and not reached by TME, could make up for the difference.

It is widespread policy that patients are subjected to postoperative radiochemotherapy if the histopathological report proves a stage UICC II or III disease in preoperatively understaged patients. However, based on the results of the CAO/ARO/AIO-94 trial [8] and the MRCCR07 trial [20], this has to be challenged. Postoperative radiotherapy causes side effects without any oncologic benefit. Its effectivity is limited and local recurrence rates are comparable to the rates achieved by TME alone.

The prognostic relevance of the circumferential margin has been well documented [29]. Taylor et al. [35] demonstrated in their study that in selected rectal cancer patients, identified by determination of the CRM and other good prognosis criteria using high-resolution MRI, local recurrence rates were as low as 3.3\%. However, it has to be clearly differentiated between the preoperative evaluation and the postoperative evaluation of the CRM by the pathologists. In case of an inadequate TME, both could be negative, though lymph node metastases within the mesorectum remained within the pelvis. This could explain the greater differences in local recurrence in the randomized trials to date in stage III rectal cancer.

\section{Conclusion}

In the treatment of patients with rectal cancer, surgery remains the mainstay. Since radiotherapy and chemoradiotherapy may improve local recurrence and survival in some patients, many patients with resectable rectal cancer are overtreated. Those patients are exposed to the risk of acute and long-term toxicity due to unnecessary radiotherapy. Subsets of patients who do not need preoperative radiotherapy need to be better defined. According to available evidence, those are patients with stage I tumors and tumors more than $12 \mathrm{~cm}$ proximal the anal verge. There is evidence that patients with a negative CRM ( $\geq 1-2 \mathrm{~mm}$ ) as assessed by preoperative MRI might not benefit from neoadjuvant treatment. This hypothesis, though, has to be proven by a randomized controlled trial.

\section{Disclosure Statement}

The authors declare that no financial or other conflict of interest exists in relation to the content of the article.

\section{References}

1 Malvezzi M, Arfe A, Bertuccio P, et al: European cancer mortality predictions for the year 2011. Ann Oncol 2011;22:947-956.

-2 Kapiteijn E, Marijnen CA, Colenbrander AC, et al: Local recurrence in patients with rectal cancer diagnosed between 1988 and 1992: a population-based study in the west Netherlands. Eur J Surg Oncol 1998;24:528-535.

-3 Phillips RK, Hittinger R, Blesovsky L, et al: Local recurrence following 'curative' surgery for large bowel cancer: II. The rectum and rectosigmoid. Br J Surg 1984;71:17-20.

4 Heald RJ, Husband EM, Ryall RD: The mesorectum in rectal cancer surgery - the clue to pelvic recurrence? Br J Surg 1982;69:613616.

5 Heald RJ, Ryall RD: Recurrence and survival after total mesorectal excision for rectal cancer. Lancet 1986;1:1479-1482.
6 Adjuvant radiotherapy for rectal cancer: a systematic overview of 8,507 patients from 22 randomised trials. Lancet 2001;358:12911304.

7 Camma C, Giunta M, Fiorica F, et al: Preoperative radiotherapy for resectable rectal cancer: a meta-analysis. JAMA 2000;284: 1008-1015.

-8 Sauer R, Becker H, Hohenberger W, et al: Preoperative versus postoperative chemoradiotherapy for rectal cancer. N Engl J Med 2004;351:1731-1740.

-9 Schmiegel W, Reinacher-Schick A, Arnold $\mathrm{D}$, et al: Update S3-guideline 'colorectal cancer' 2008 (in German). Z Gastroenterol 2008; 46:799-840.

10 Cawthorn SJ, Parums DV, Gibbs NM, et al: Extent of mesorectal spread and involvement of lateral resection margin as prognostic factors after surgery for rectal cancer. Lancet 1990;335:1055-1059.
11 Merkel S, Mansmann U, Siassi M, et al: The prognostic inhomogeneity in $\mathrm{pT} 3$ rectal carcinomas. Int J Colorectal Dis 2001;16:298304.

12 Willett CG, Badizadegan K, Ancukiewicz M, et al: Prognostic factors in stage T3N0 rectal cancer: do all patients require postoperative pelvic irradiation and chemotherapy? Dis Colon Rectum 1999;42:167-173.

13 Bruheim K, Svartberg J, Carlsen E, et al: Radiotherapy for rectal cancer is associated with reduced serum testosterone and increased FSH and LH. Int J Radiat Oncol Biol Phys 2008;70:722-727.

14 Bruheim K, Tveit KM, Skovlund E, et al: Sexual function in females after radiotherapy for rectal cancer. Acta Oncol 2010;49:826832 . 
15 Bruheim K, Guren MG, Dahl AA, et al: Sexual function in males after radiotherapy for rectal cancer. Int J Radiat Oncol Biol Phys 2010;76:1012-1017.

- 16 Bruheim K, Guren MG, Skovlund E, et al: Late side effects and quality of life after radiotherapy for rectal cancer. Int J Radiat Oncol Biol Phys 2010;76:1005-1011.

17 Peeters KC, van de Velde CJ, Leer JW, et al: Late side effects of short-course preoperative radiotherapy combined with total mesorectal excision for rectal cancer: increased bowel dysfunction in irradiated patients - a Dutch colorectal cancer group study. J Clin Oncol 2005;23:6199-6206.

- 18 van Gijn W, Marijnen CA, Nagtegaal ID, et al: Preoperative radiotherapy combined with total mesorectal excision for resectable rectal cancer: 12-year follow-up of the multicentre, randomised controlled TME trial. Lancet Oncol 2011;12:575-582.

$\checkmark 19$ Nagtegaal ID, Marijnen CA, Kranenbarg EK, et al: Circumferential margin involvement is still an important predictor of local recurrence in rectal carcinoma: not one millimeter but two millimeters is the limit. Am J Surg Pathol 2002;26:350-357.

20 Sebag-Montefiore D, Stephens RJ, Steele R, et al: Preoperative radiotherapy versus selective postoperative chemoradiotherapy in patients with rectal cancer (MRC CR07 and NCIC-CTG C016): a multicentre, randomised trial. Lancet 2009;373:811-820.

21 Enker WE, Thaler HT, Cranor ML, et al: Total mesorectal excision in the operative treatment of carcinoma of the rectum. J Am Coll Surg 1995;181:335-346.
22 Kapiteijn E, Putter H, van de Velde CJ: Impact of the introduction and training of total mesorectal excision on recurrence and survival in rectal cancer in the Netherlands. $\mathrm{Br}$ J Surg 2002;89:1142-1149.

23 Martling A, Holm T, Rutqvist LE, et al: Impact of a surgical training programme on rectal cancer outcomes in Stockholm. Br J Surg 2005;92:225-229.

24 Wibe A, Moller B, Norstein J, et al: A national strategic change in treatment policy for rectal cancer - implementation of total mesorectal excision as routine treatment in Norway. A national audit. Dis Colon Rectum 2002;45:857-866.

25 Quirke P, Steele R, Monson J, et al: Effect of the plane of surgery achieved on local recurrence in patients with operable rectal cancer: a prospective study using data from the MRC CR07 and NCIC-CTG CO16 randomised clinical trial. Lancet 2009;373:821-828.

26 Folkesson J, Birgisson H, Pahlman L, et al: Swedish Rectal Cancer Trial: long lasting benefits from radiotherapy on survival and local recurrence rate. J Clin Oncol 2005;23: 5644-5650.

27 Hermanek P, Merkel S, Fietkau R, et al: Regional lymph node metastasis and locoregional recurrence of rectal carcinoma in the era of TME (corrected) surgery. Implications for treatment decisions. Int J Colorectal Dis 2010;25:359-368.

28 Extramural depth of tumor invasion at thinsection MR in patients with rectal cancer: results of the MERCURY study. Radiology 2007;243:132-139.

29 Nagtegaal ID, Quirke P: What is the role for the circumferential margin in the modern treatment of rectal cancer? J Clin Oncol 2008;26:303-312.

30 Quirke P, Durdey P, Dixon MF, et al: Local recurrence of rectal adenocarcinoma due to inadequate surgical resection. Histopathological study of lateral tumour spread and surgical excision. Lancet 1986;2:996-999.
31 Harrison JC, Dean PJ, el-Zeky F, et al: From Dukes through Jass: pathological prognostic indicators in rectal cancer. Hum Pathol 1994;25:498-505.

32 Jass JR, Atkin WS, Cuzick J, et al: The grading of rectal cancer: historical perspectives and a multivariate analysis of 447 cases. Histopathology 1986;10:437-459.

-33 Shepherd NA, Baxter KJ, Love SB: Influence of local peritoneal involvement on pelvic recurrence and prognosis in rectal cancer. J Clin Pathol 1995;48:849-855.

34 Simunovic M, Sexton R, Rempel E, et al: Optimal preoperative assessment and surgery for rectal cancer may greatly limit the need for radiotherapy. Br J Surg 2003;90:9991003.

35 Taylor FG, Quirke P, Heald RJ, et al: Preoperative high-resolution magnetic resonance imaging can identify good prognosis stage I, II, and III rectal cancer best managed by surgery alone: a prospective, multicenter, European study. Ann Surg 2011;253:711-719.

36 Frasson M, Garcia-Granero E, Roda D, et al: Preoperative chemoradiation may not always be needed for patients with T3 and T2N+ rectal cancer. Cancer 2011;117:31183125.

37 Improved survival with preoperative radiotherapy in resectable rectal cancer. Swedish Rectal Cancer Trial. N Engl J Med 1997;336: 980-987.

38 Cecil TD, Sexton R, Moran BJ, et al: Total mesorectal excision results in low local recurrence rates in lymph node-positive rectal cancer. Dis Colon Rectum 2004;47:11451149. 